\title{
Model Discrimination in Chemical Kinetics
}

\author{
Burcu Özdemir and Selahattin Gültekin*
}

Doğuş University, Acıbadem, Kadıköy 34722, Istanbul, Turkey

\begin{abstract}
In studies on chemical kinetics, generally after the rate data have been taken, a mechanism and an associated rate law model are proposed based on the data taken. Frequently, more than one mechanism and rate law may be consistent with data. In order to find the correct rate law, regression techniques (model discrimination) are applied to indentify which model equation best fits the data by choosing the one with the smaller sum of squares. With this non-linear regression technique, rate parameters with $95 \%$ confidence limits are calculated along with residues. Of course, model parameters must be realistic. For example, reaction rate constants, activation energies or adsorption equilibrium constants must be positive by comparing the calculated value of parameters with $95 \%$ confidence limits, one can judge about the validity of the model.

In this paper, model discrimination will be applied to certain data from the literature along with the suggested heterogeneous catalytic models such as Langmuir-Hinshelwood Kinetic Model or Rideal-Eley Model.

A reaction of $A+B \rightarrow C+D$ type have been selected (like methanation) with the rate laws given below:

$-r_{A}=k P_{A} P_{B} /\left(1+K_{A} P_{A}+K_{B} P_{B}+K_{C} P_{C}+K_{D} P_{D}\right)^{2} \quad$ dual-site Langmuir-Hinshelwood Model

$-r_{A}=k P_{A} P_{B} /\left(1+K_{A} P_{A}+K_{B} P_{B}+K_{C} P_{C}+K_{D} P_{D}\right) \quad$ single-site Langmuir-Hinshelwood Model

$-r_{A}=k P_{A} P_{B} /\left(1+K_{A} P_{A}+K_{B} P_{B}\right)^{2} \quad$ only reactants are adsorbed

$-r_{A}=k P_{A} P_{B} /\left(1+K_{A} P_{A}\right)^{2} \quad$ dual-site Rideal-Eley Model

$-r_{A}=k P_{A}^{a} \cdot P_{B}^{b} \quad$ power-law
\end{abstract}

In this study,

$$
\mathrm{CO}+3 \mathrm{H}_{2} \rightarrow \mathrm{CH}_{4}+\mathrm{H}_{2} \mathrm{O}
$$

reaction rate data were tested for five different models to find the most suitable rate expression by model discrimination method taking the advantage of powerful POLYMATH package program.

\section{INTRODUCTION}

\section{Regression Techniques}

In empirical studies, in order to determine the parameters for the postulated model, very powerful regression techniques (methods) are used.

Regression methods basically are as follows [1]

a) Linear regression (like $y=a x+b$ ), where a and $\mathrm{b}$ are to be determined.

b) Multiple regression (like $y=a_{1} x_{1}+a_{2} x_{2}+\ldots .+a_{n} x_{n}$ ), where $a_{i}$ 's are parameters to be determined.

c) Polynomial regression (like

$\left.y=a_{n} x^{n}+a_{n-1} x^{n-1}+\ldots+a_{1} x+a_{0}\right), a_{i}$ 's are parameter to be determined from regression.

*Address correspondence to this author at the Doğuş University, Acıbadem, Kadıköy 34722, Istanbul, Turkey; E-mail: sgultekin@dogus.edu.tr

\section{d) Non-linear regression [2].}

This is very common and can be used almost under any condition. General form is $y=f\left(x_{1}, x_{2}, \ldots, x_{n}, a_{1}, a_{2}, \ldots, a_{n}\right)$, where $\mathrm{n}=\#$ of experiments, $\mathrm{m}=\#$ of parameters to be determined providing $n>\mathrm{m}+1$.

The common features of the above regression techniques are that it is to make the variance minimum, and to make the correction factor as close to unity as possible. After determination of parameters, the next step is to check to see whether they are physically meaningful or not. For example, if adsorption equilibrium constant is one of the parameters, then it must decrease with increasing temperature as adsorption is an exothermic process (Le Chatelier Principle)

In kinetic studies, generally one faces very complicated rate expressions on heterogeneous catalysts. Those rate expressions may obey Langmuir-Hinshelwood or Rideal-Eley model $[3,4]$.

In general, if we have a reaction of $A+B \rightarrow C$ we may have possible rate expressions as 
$-r_{A}=\frac{k P_{A} P_{B}}{\left(1+K_{A} P_{A}+K_{B} P_{B}+K_{C} P_{C}\right)^{2}}$ (Dual-site model)

$-r_{A}=\frac{k P_{A} P_{B}}{\left(1+K_{A} P_{A}+K_{B} P_{B}+K_{C} P_{C}\right.}($ Single-site model $)$

$-r_{A}=\frac{k P_{A} P_{B}}{\left(1+K_{A} P_{A}\right)^{2}}($ Dual-site, Rideal-Eley $)$

$-r_{A}=k P_{A}^{a} P_{B}^{b}$ (Apparent power-law expression)

In kinetic studies, for example, one may have three different mechanisms and three different rate-determining steps. Therefore, one will have nine different rate expressions. In order to determine the correct rate expression, model discrimination method is being used. The essence of this method is not only to minimize the variance, but also to keep the correction factor as close to one (unity) as possible. In addition to the above two criteria, one also has to check the physical validity of the determined parameters.

\section{RESULTS}

Now we will chose the reaction of methanation [4] on $\mathrm{Ni}$ catalyst where initial rates are taken at constant temperature at variety partial pressures of reactants and products.

Consider the reaction

$$
\mathrm{CO}+3 \mathrm{H}_{2} \rightarrow \mathrm{CH}_{4}+\mathrm{H}_{2} \mathrm{O} \quad \text { (methanation) }
$$

Assuming the following runs were carried out under the given conditions ( see Table 1).

Table 1. Initial Rates Obtained at Various Partial Pressures

\begin{tabular}{|c|c|c|c|c|c|}
\hline Run \# & $\mathbf{r}_{\mathbf{A}} *$ & $\mathbf{P}_{\mathbf{C O}}{ }^{* *}$ & $\mathbf{P}_{\mathbf{H} 2} * *$ & $\mathbf{P}_{\mathbf{H} 2 \mathbf{O}^{* *}}$ & $\mathbf{P}_{\mathbf{C H} 4}{ }^{* *}$ \\
\hline \hline $\mathbf{1}$ & 0.1219 & 1 & 1 & 0 & 0 \\
\hline $\mathbf{2}$ & 0.0944 & 1 & 1 & 1 & 1 \\
\hline $\mathbf{3}$ & 0.0943 & 1 & 1 & 1 & 2 \\
\hline $\mathbf{4}$ & 0.0753 & 1 & 1 & 2 & 1 \\
\hline $\mathbf{5}$ & 0.0753 & 1 & 1 & 2 & 2 \\
\hline $\mathbf{6}$ & 0.0512 & 1 & 1 & 4 & 1 \\
\hline $\mathbf{7}$ & 0.0280 & 1 & 1 & 8 & 1 \\
\hline $\mathbf{8}$ & 0.1274 & 1 & 2 & 1 & 1 \\
\hline $\mathbf{9}$ & 0.1056 & 1 & 2 & 2 & 2 \\
\hline $\mathbf{1 0}$ & 0.1203 & 1 & 4 & 2 & 2 \\
\hline $\mathbf{1 1}$ & 0.1189 & 1 & 8 & 1 & 1 \\
\hline $\mathbf{1 2}$ & 0.0782 & 2 & 1 & 1 & 1 \\
\hline $\mathbf{1 3}$ & 0.1204 & 2 & 2 & 1 & 1 \\
\hline $\mathbf{1 4}$ & 0.1057 & 2 & 2 & 2 & 0 \\
\hline $\mathbf{1 5}$ & 0.1056 & 2 & 2 & 2 & 1 \\
\hline $\mathbf{1 6}$ & 0.1056 & 2 & 2 & 2 & 2 \\
\hline $\mathbf{1 7}$ & 0.1552 & 2 & 4 & 1 & 1 \\
\hline $\mathbf{1 8}$ & 0.0533 & 4 & 1 & 1 & 1 \\
\hline $\mathbf{1 9}$ & 0.0911 & 4 & 2 & 1 & 1 \\
\hline $\mathbf{2 0}$ & 0.0317 & 8 & 1 & 1 & 1 \\
\hline $\mathbf{2 1}$ & 0.1476 & 8 & 8 & 1 & 1 \\
\hline
\end{tabular}

${ }^{*} \mathrm{r}_{\mathrm{A}}[\mathrm{mole} / \mathrm{kg}$. cat.s $],{ }^{* *} \mathrm{P}_{\mathrm{i}}[\mathrm{atm}]$.
We will consider the following plausible rate expressions and by model discrimination we will reach hopefully to the true rate expression.

a) All reactants and products are adsorbed with dual-site mechanism:

$$
-r_{A}=\frac{k P_{C O} \cdot P_{H_{2}}}{\left(1+K_{C O} \cdot P_{C O}+K_{H_{2}} \cdot P_{H_{2}}+K_{\mathrm{H}_{2} O} \cdot P_{H_{2} \mathrm{O}}+K_{C_{4}} P_{C_{4}}\right)^{2}}
$$

(Dual-site)

b) All reactants and products are adsorbed with singlesite mechanism:

$$
-r_{A}=\frac{k P_{C O} \cdot P_{\mathrm{H}_{2}}}{\left(1+K_{C O} \cdot P_{C O}+K_{\mathrm{H}_{2}} \cdot P_{\mathrm{H}_{2}}+K_{\mathrm{H}_{2} \mathrm{O}} \cdot P_{\mathrm{H}_{2} \mathrm{O}}+K_{\mathrm{CH}_{4}} P_{\mathrm{CH}_{4}}\right)}
$$

(Single-site)

c) Only reactants are adsorbed with dual-site mechanism:

$$
-r_{A}=\frac{k P_{C O} \cdot P_{H_{2}}}{\left(1+K_{C O} \cdot P_{C O}+K_{\mathrm{H}_{2}} P_{\mathrm{H}_{2}}\right)^{2}} \quad \text { (Only reactants are }
$$
adsorbed, dual-site)

d) Only $\mathrm{CO}$ is adsorbed; $\mathrm{H}_{2}$ comes directly from gas phase and reacts with adsorbed $\mathrm{CO}$ (Rideal-Eley Mechanism)

$$
-r_{A}=\frac{k P_{C O} \cdot P_{H_{2}}}{\left(1+K_{C O} \cdot P_{C O}\right)^{2}} \text { (Rideal-Eley, dual-site) }
$$

e) A power-law expression:

$$
-r_{A}=k \cdot P_{C O}^{a} \cdot P_{H_{2}}^{b} \text { (Power-Law) }
$$

In the following pages, these five different models were tried on Polymath ${ }^{\circledR}$ program. The results are self explanatory, and are given in Figs. (1-5).

\section{Model a:}

$$
-r_{A}=\frac{k P_{C O} \cdot P_{H_{2}}}{\left(1+K_{C O} \cdot P_{C O}+K_{\mathrm{H}_{2}} \cdot P_{\mathrm{H}_{2}}+K_{\mathrm{H}_{2} \mathrm{O}} \cdot P_{\mathrm{H}_{2} \mathrm{O}}+K_{\mathrm{CH}_{4}} P_{\mathrm{CH}_{4}}\right)^{2}}
$$

\section{Output of Model a:}

$r_{A}=k P_{C O} P_{H} /\left(1+K_{C O} P_{C O}+K_{H} P_{H}+K_{\mathrm{H}_{2} \mathrm{O}} P_{\mathrm{H}_{2} \mathrm{O}}+K_{\mathrm{CH}_{4}} P_{\mathrm{CH}_{4}}\right)^{2}$

\section{POLYMATH Results}

Nonlinear Regression (L-M)

\begin{tabular}{|c|c|c|c|}
\hline Variable & Ini. Guess & Value & 95\% Confidence \\
\hline $\mathbf{k}$ & 4 & 7.9628870 & 0.0367133 \\
\hline $\mathbf{K C O}$ & 2 & 5.0877643 & 0.0124837 \\
\hline $\mathbf{K H}$ & 4 & 1.9950241 & 0.0047754 \\
\hline $\mathbf{K H}_{\mathbf{2}} \mathbf{O}$ & 5 & 1.0969188 & 0.0030085 \\
\hline $\mathbf{K C H}_{\mathbf{4}}$ & 1 & 0.0046140 & 0.0015179 \\
\hline
\end{tabular}

Nonlinear regression settings.

$\operatorname{Max} \#$ iterations $=64$ 


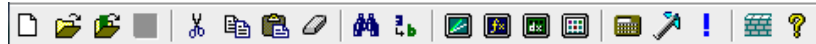

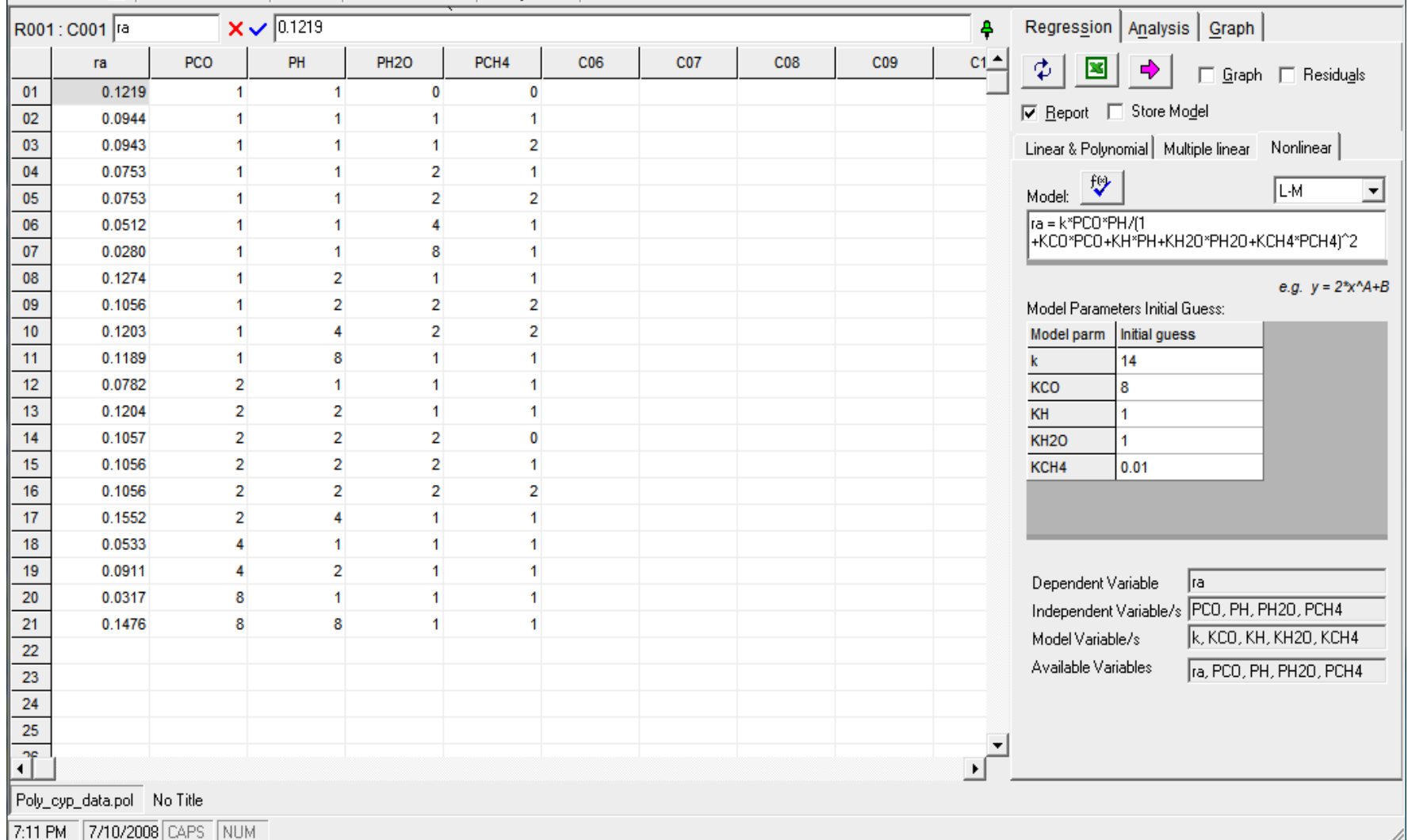

Fig. (1). Input of the data in POLYMATH Screen for Model a.

\begin{tabular}{|c|c|c|c|c|c|c|c|c|c|c|c|c|c|c|c|}
\hline (2) PC & ATH 6.10 & ducatio & al $\operatorname{Re}$ & ase - [ & ITable] & $=-5$ & & 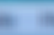 & & & & & & & b|回|x \\
\hline 圖 F & Program & Edit & ow & olumn & ormat & sis Exa & le & Wind & & & & & & & \begin{tabular}{|l|l|}
- & 5 \\
\end{tabular} \\
\hline $0 E$ & a & 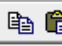 & $\theta$ & की & 国国 & 䔬|慖 & 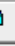 & 置 & & & & & & & \\
\hline R001 & $1 0 1 \longdiv { \text { ra } }$ & & $x \vee$ & 0.1219 & & & & & & & & \& & Regression & Analysis | Graph | & \\
\hline & ra & PCO & t & $\mathrm{PH}$ & PH2O & $\mathrm{PCH} 4$ & & $\mathrm{C} 06$ & $\mathrm{C} 07$ & $\mathrm{cos}$ & $\operatorname{cog}$ & c1 $\Delta$ & $\rightarrow$ 圆 & $\Rightarrow 1-$ Graph & Г Residuals \\
\hline 01 & 0.1219 & & 1 & & & & & & & & & & -1 & & \\
\hline 02 & 0.0944 & & 1 & & & & & & & & & & $\sqrt{\nabla}$ Report $\Gamma$ & Store Model & \\
\hline 03 & 0.0943 & & 1 & & & & & & & & & & Linear \& Polyno & omial| Multiple linear & Nonlinear| \\
\hline 04 & 0.0753 & & 1 & & & & & & & & & & $f(x)$ & & \\
\hline 05 & 0.0753 & & 1 & & & & & & & & & & Model: & & L-M \\
\hline 06 & 0.0512 & & 1 & & & & & & & & & & $r a=k^{*} P C O \times P h$ & $\mathrm{H} /(1$ & \\
\hline 07 & 0.0280 & & 1 & & & & & & & & & & ${ }^{\mathrm{PCO}}+\mathrm{K}$ & $\mathrm{KC}$ & 14) \\
\hline 08 & 0.1274 & & 1 & & & & & & & & & & & & e.g. $y=2^{2} x^{\wedge} A+B$ \\
\hline 09 & 0.1056 & & 1 & & & & & & & & & & Model Paramel & ters Initial Guess: & \\
\hline 10 & 0.1203 & & 1 & & & & & & & & & & Model parm & Initial guess & \\
\hline 11 & 0.1189 & & 1 & & & & & & & & & & $\mathrm{k}$ & 14 & \\
\hline 12 & 0.0782 & & 2 & & & & & & & & & & $\mathrm{KCO}$ & 8 & \\
\hline 13 & 0.1204 & & 2 & & & & & & & & & & $\mathrm{KH}$ & 1 & \\
\hline 14 & 0.1057 & & 2 & & & & & & & & & & $\mathrm{KH} 2 \mathrm{O}$ & 1 & \\
\hline 15 & 0.1056 & & 2 & & & & & & & & & & $\mathrm{KCH} 4$ & 0.01 & \\
\hline 16 & 0.1056 & & 2 & & & & & & & & & & & & \\
\hline 17 & 0.1552 & & 2 & & & & & & & & & & & & \\
\hline 18 & 0.0533 & & 4 & & & & & & & & & & & & \\
\hline 19 & 0.0911 & & 4 & & & & & & & & & & Dependent $V$ & ariable ra & \\
\hline 20 & 0.0317 & & 8 & & & & & & & & & & Independent: & Variable/s $\mathrm{PCO}, \mathrm{PH}, \mathrm{PH}$ & $\mathrm{H} 2 \mathrm{O}, \mathrm{PCH} 4$ \\
\hline 21 & 0.1476 & & 8 & & & & & & & & & & Model Variable & $\mathrm{k}, \mathrm{KCO}, \mathrm{KH}$, & $\mathrm{H}, \mathrm{KH} 20, \mathrm{KCH} 4$ \\
\hline$\frac{22}{23}$ & & & & & & & & & & & & & Available Vari & ra, PCO, PH & $\mathrm{H}, \mathrm{PH} 2 \mathrm{O}, \mathrm{PCH} 4$ \\
\hline$\frac{23}{24}$ & & & & & & & & & & & & & & & \\
\hline 25 & & & & & & & & & & & & & & & \\
\hline 10 & & & & & & & & & & & & 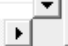 & & & \\
\hline Poly_ & data_sing|c & No & & & & & & & & & & & & & \\
\hline $7: 26 \mathrm{P}$ & 7/10/200 & PSS & NUM & & & & & & & & & & & & \\
\hline
\end{tabular}

Fig. (2). Input of the data in POLYMATH Screen for Model b. 
Precision

$\begin{array}{ll}\mathbf{R}^{\wedge} \mathbf{2} & =0.9999995 \\ \mathbf{R}^{\wedge} \mathbf{2 a d j} & =0.9999993 \\ \text { Rmsd } & =5.321 \mathrm{E}-06 \\ \text { Variance } & =7.804 \mathrm{E}-10\end{array}$

\section{Model b:}

$-r_{A}=\frac{k P_{C O} \cdot P_{\mathrm{H}_{2}}}{\left(1+K_{\mathrm{CO}} \cdot P_{\mathrm{CO}}+K_{\mathrm{H}_{2}} \cdot P_{\mathrm{H}_{2}}+K_{\mathrm{H}_{2} \mathrm{O}} \cdot P_{\mathrm{H}_{2} \mathrm{O}}+K_{\mathrm{CH}_{4}} P_{\mathrm{CH}_{4}}\right)}$

\section{Output of Model b:}

$r_{A}=k P_{C O} P_{H} /\left(1+K_{C O} P_{C O}+K_{H} P_{H}+K_{\mathrm{H}_{2} \mathrm{O}} P_{\mathrm{H}_{2} \mathrm{O}}+K_{C_{4}} P_{C_{4}}\right)^{2}$

\begin{tabular}{|c|c|c|c|}
\hline Variable & Ini guess & Value & $\mathbf{9 5 \%}$ Confidence \\
\hline $\mathbf{k}$ & 14.0 & 2.747922 & 0.0023620 \\
\hline $\mathbf{K C O}$ & 8.0 & 51.41168 & 0.0593556 \\
\hline $\mathbf{K H}$ & 1.0 & 12.89503 & 0.0368865 \\
\hline $\mathbf{K H}_{\mathbf{2}} \mathbf{O}$ & 1.0 & 2.123223 & 0.0692380 \\
\hline $\mathbf{K C H}_{4}$ & 0.01 & -1.989895 & 0.0982922 \\
\hline
\end{tabular}

Nonlinear regression settings

Max \# iterations $=64$

Precision

$$
\begin{array}{ll}
\mathbf{R}^{\wedge} \mathbf{2} & =-1.586834 \\
\mathbf{R}^{\wedge} \mathbf{2 a d j} & =-2.233542 \\
\text { Rmsd } & =0.011789 \\
\text { Variance } & =0.0038306 \\
\text { Model c: }-r_{\mathrm{A}}=\frac{k P_{\mathrm{CO}} \cdot P_{\mathrm{H}_{2}}}{\left(1+K_{\mathrm{CO}} \cdot P_{\mathrm{CO}}+K_{\mathrm{H}_{2}} P_{\mathrm{H}_{2}}\right)^{2}}
\end{array}
$$

Output of Model c: $r_{A}=k P_{C O} P_{H} /\left(1+K_{C O} P_{C O}+K_{H} P_{H}\right)^{2}$

\begin{tabular}{|c|c|c|c|c|c|c|c|c|c|c|c|c|c|c|}
\hline (20) $\mathrm{PC}$ & ATH 6.10 & ducation & nal Relc & ase - & ta Table] & sin in & 40 & & & & & & & 可回 $x$ \\
\hline 䧃 & Program & Edit $R$ & Row & Columr & Format A & ysis Exa & ples Wind & Help & & & & & & $-5 x$ \\
\hline 08 & 301 & 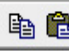 & 0 & A & 回 国 & 国|国 & ！囬 & & & & & & & \\
\hline Roo & $0 1 \longdiv { \text { ra } }$ & & $x v$ & 0.1219 & & & & & & & कి & Regression & |Analysis $|\underline{G r a p h}|$ & \\
\hline & ra & $\mathrm{PCO}$ & 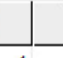 & $\mathrm{PH}$ & PH2O & $\mathrm{PCH}_{4}$ & $\mathrm{C} 06$ & $\mathrm{CO7}$ & $\cos$ & $\operatorname{cog}$ & C1 1 & 国 & $\Leftrightarrow \Gamma$ Graph & $\ulcorner$ Residuals \\
\hline 01 & 0.1219 & & 1 & & & & 0 & & & & & $\nabla$ Report & Store Model & \\
\hline 02 & 0.0944 & & 1 & & & & 1 & & & & & & & \\
\hline 03 & 0.0943 & & 1 & & & & 2 & & & & & Linear \& Polyno & omial| Multiple linear & Nonlinear \\
\hline 04 & 0.0753 & & 1 & & & & 1 & & & & & & & \\
\hline 05 & 0.0753 & & 1 & & & & 2 & & & & & Model: & & L-M \\
\hline 06 & 0.0512 & & 1 & & & & 1 & & & & & $r a=k^{*} P C O * P F$ & $\mathrm{H} /(1+\mathrm{KCO} \times \mathrm{PCO}+\mathrm{KH} \times \mathrm{PH}$ & $H)^{\wedge} 2$ \\
\hline 07 & 0.0280 & & 1 & & & & 1 & & & & & & & \\
\hline 08 & 0.1274 & & 1 & & & & 1 & & & & & & & e.g. $y=2^{2} x^{\wedge} A+B$ \\
\hline 09 & 0.1056 & & 1 & & & & 2 & & & & & Model Paramet & ters Initial Guess: & \\
\hline 10 & 0.1203 & & 1 & & & & 2 & & & & & Model parm & Initial guess & \\
\hline 11 & 0.1189 & & 1 & & & & 1 & & & & & $k$ & 14 & \\
\hline 12 & 0.0782 & & 2 & & & & 1 & & & & & $\mathrm{KCO}$ & 8 & \\
\hline 13 & 0.1204 & & 2 & & & & 1 & & & & & $\overline{\mathrm{KH}}$ & 1 & \\
\hline 14 & 0.1057 & & 2 & & & & 0 & & & & & & & \\
\hline 15 & 0.1056 & & 2 & & & & 1 & & & & & & & \\
\hline 16 & 0.1056 & & 2 & & & & 2 & & & & & & & \\
\hline 17 & 0.1552 & & 2 & & & & 1 & & & & & & & \\
\hline 18 & 0.0533 & & 4 & & & & 1 & & & & & & & \\
\hline 19 & 0.0911 & & 4 & & & & 1 & & & & & Dependent Va & 'ariable $\quad$ ra & \\
\hline 20 & 0.0317 & & 8 & & & & 1 & & & & & Independent: & Variable/s $\mathrm{PCO}, \mathrm{PH}$ & \\
\hline 21 & 0.1476 & & 8 & & & & 1 & & & & & Model Variable & $\mathrm{le} / \mathrm{s} \quad \mathrm{k}, \mathrm{KCO}, \mathrm{KH}$ & \\
\hline 22 & & & & & & & & & & & & inver valiane & 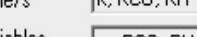 & \\
\hline 23 & & & & & & & & & & & & & ra, $\mathrm{PCO}, \mathrm{PH}$ & $\mathrm{H}, \mathrm{PH} 2 \mathrm{O}, \mathrm{PCH} 4$ \\
\hline 24 & & & & & & & & & & & & & & \\
\hline
\end{tabular}

\begin{tabular}{|c|c|c|c|}
\hline Variable & Ini guess & Value & $\mathbf{9 5 \%}$ Confidence \\
\hline $\mathbf{k}$ & 14.0 & 0.7700806 & 0.6088063 \\
\hline KCO & 8.0 & 1.540737 & 0.7859312 \\
\hline KH & 1.0 & 0.5896616 & 0.3142481 \\
\hline
\end{tabular}

Precision

$\begin{array}{ll}\mathbf{R}^{\wedge} \mathbf{2} & =0.7311287 \\ \mathbf{R}^{\wedge} \mathbf{2 a d j} & =0.7012541 \\ \mathbf{R m s d} & =0.0038007 \\ \text { Variance } & =0.0003539\end{array}$

Model d: $-r_{A}=\frac{k P_{C O} \cdot P_{H_{2}}}{\left(1+K_{C O} \cdot P_{C O}\right)^{2}}$

Fig. (3). Input of the data in POLYMATH Screen for Model c. 


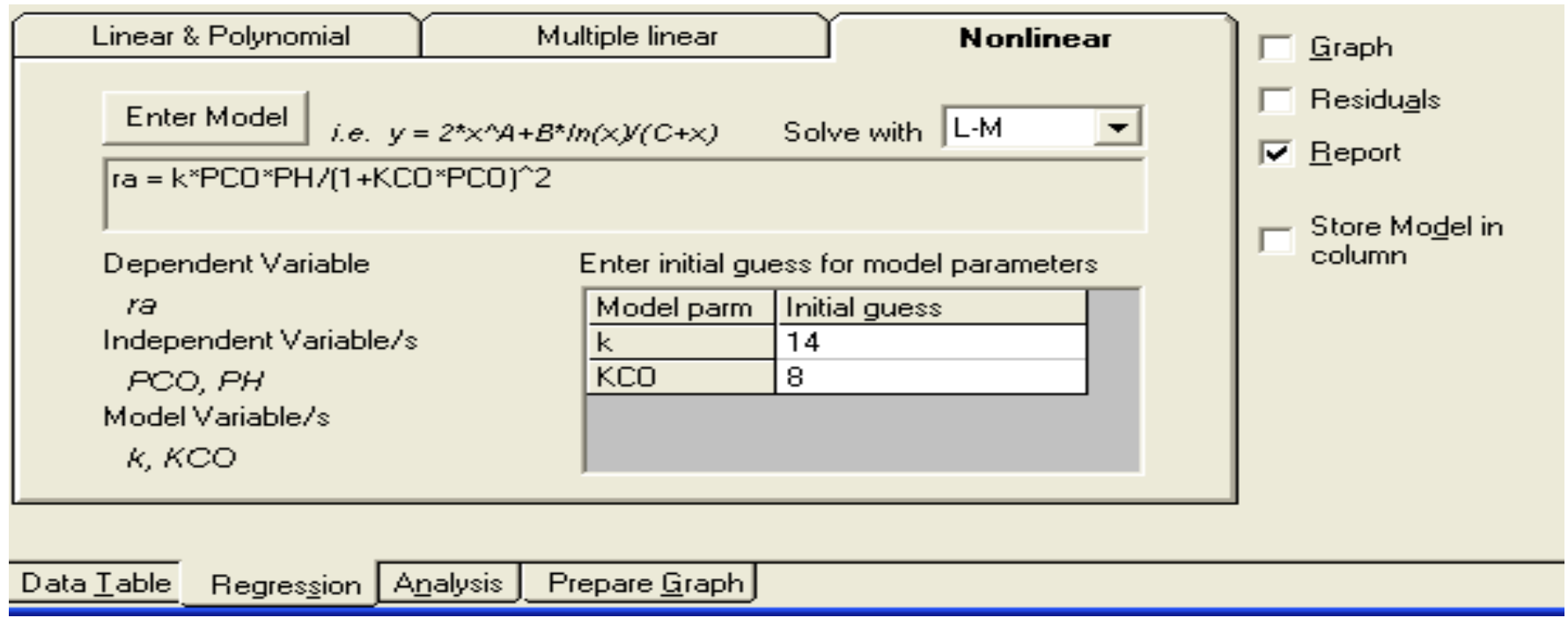

Fig. (4). Input of the data in POLYMATH Screen for Model d.

Output of Model d: $r_{A}=k P_{C O} P_{H} /\left(1+K_{C O} P_{C O}\right)^{2}$

\begin{tabular}{|c|c|c|c|}
\hline Variable & Ini Guess & Value & 95\% Confidence \\
\hline $\mathbf{k}$ & 14.0 & 0.062234 & $5.47 \mathrm{E}-06$ \\
\hline KCO & 8.0 & 0.453103 & $3.977 \mathrm{E}-05$ \\
\hline
\end{tabular}

Nonlinear regression settings.

Max \# iterations $=64$
Precision

$\begin{array}{ll}\mathbf{R}^{\wedge} \mathbf{2} & =-1.282797 \\ \mathbf{R}^{\wedge} \mathbf{2 a d j} & =-1.402944 \\ \mathbf{R} \mathbf{m s d} & =0.0110745 \\ \text { Variance } & =0.0028467\end{array}$

Model e: $-r_{A}=k \cdot P_{C O}^{a} \cdot P_{H_{2}}^{b}$

Output of Model e: $\mathrm{ra}=\mathrm{k}^{*} \mathrm{PCO}^{\wedge} \mathrm{a}^{*} \mathrm{PH}^{\wedge} \mathrm{b}$

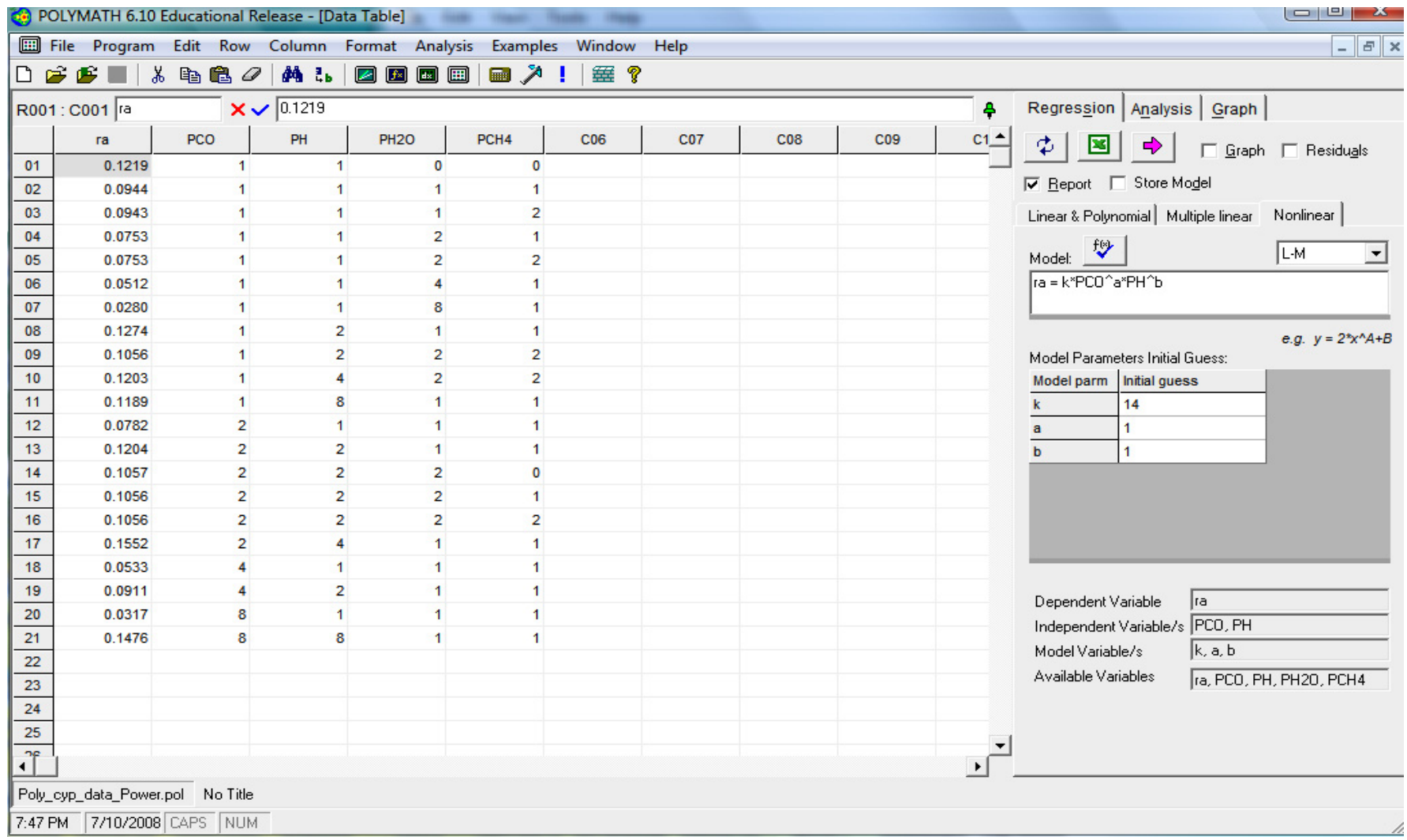

Fig. (5). Input of the data in POLYMATH Screen for Model e. 


\begin{tabular}{|c|c|c|c|}
\hline Variable & Ini Guess & Value & 95\% Confidence \\
\hline k & 14.0 & 0.0806184 & $3.076 \mathrm{E}-06$ \\
\hline a & 1.0 & -0.0554411 & $4.271 \mathrm{E}-05$ \\
\hline b & 1.0 & 0.3205555 & $3.242 \mathrm{E}-05$ \\
\hline
\end{tabular}

Nonlinear regression settings.

Max \# iterations $=64$

Precision

$\begin{array}{ll}\mathbf{R}^{\wedge} \mathbf{2} & =0.4902824 \\ \mathbf{R}^{\wedge} \mathbf{2 a d j} & =0.4336471 \\ \mathbf{R m s d} & =0.0052331 \\ \text { Variance } & =6.709 \mathrm{E}-04\end{array}$

When variance, correction factor (coefficient) and the physical meaningfulness of the parameters are considered, one can clearly see that the model a fits the data in a perfect manner. In the remaining other models (i.e. b, c, d and e) either because of variance or correction factor or $95 \%$ confidence interval or combination thereof, the models seem to be inadequate and as a result they are eliminated.

\section{CONCLUSIONS}

No matter how the chemical kinetic expressions are complicated, either linear, multiple linear, non-linear or polynomial, one can find a satisfactory rate expression by means of model discrimination method. This discrimination processes are eased especially after the advent of powerful ready package programs such as POLYMATH, MATLAB, MATHCAD [5], and others. One, still, has to be very cautious in that finding the satisfactory rate expression does not mean the true (absolute) rate expression and mechanism are found [6].

\section{ACKNOWLEDGEMENTS}

Authors would like to express their thanks to Doğuş University for its financial support.

\section{REFERENCES}

[1] Polymath 6.1 User Guide, 2006.

[2] MATLAB R2006b Mathworks' Users Manual, 2006.

[3] Fogler, H.S. Elements of Chemical Reaction Engineering, 4/E, Prentice-Hall, 2006.

[4] Satterfield, C.N. Industrial Heterogeneous Catalytic Processes, 2/E, McGraw-Hill, 1990.

[5] Gültekin, S. Kimya Mühendisliğinde MATHCAD Kullanımı (Usage of MATHCAD in Chemical Engineering), Lecture Notes, Yildız Technical University, 1997.

[6] Cutlip, M.B.; Shacham, M. Problem Solving in Chemical and Biochemical Engineering with POLYMATH, Excel and MATLAB, 2/E, Prentice-Hall, 2008. 